\title{
Smoking and oxidised high-density lipoprotein: a preliminary report
}

Kazuhiko Kotani ${ }^{1}$, Kouichi Miura²

${ }^{1}$ Division of Community and Family Medicine, Jichi Medical University, Shimotsuke-City, Japan

2Division of Gastrenterology, Jichi Medical University, Shimotsuke-City, Japan

Submitted: 2 January 2021

Accepted: 6 January 2021

Arch Med Sci Atheroscler Dis 2021; 6: e28-e29

DOI: https://doi.org/10.5114/amsad.2021.105253

Copyright @ 2021 Termedia \& Banach

Cigarette smoking is a risk factor for cardiovascular disease [1]. While various mechanisms are involved in this association, the reduction in the circulating level of high-density lipoprotein (HDL), a protective factor against cardiovascular disease, by smoking is well known [1]. Furthermore, smoking is a source of oxidants, leading to oxidative modification of in vivo molecules [1], and HDL is modified oxidatively as oxidised $\mathrm{HDL}$ (oxHDL) [2]. Because oxHDL lacks the native HDL function (i.e. suppression of the antioxidative function of apolipoprotein A-I, inhibition of ABCA1-dependent cholesterol efflux from atheromatous lesions) [1-3], the oxHDL behaviour may explain the association among smoking, HDL and cardiovascular disease.

At present, information concerning the relationship between oxHDL and smoking habits is limited. Although previous studies have not directly examined oxHDL itself, the antioxidant ability of $\mathrm{HDL}$ in smokers has been reported to be impaired [4, 5]. The blood level of oxidised lipids of HDL was reportedly low in young smokers (possibly due to the active removal of smoking-induced oxidants) [6]. Accordingly, the oxHDL levels in relation to smoking should be further examined.

The present study therefore investigated the oxHDL levels in smokers. A total of 90 asymptomatic subjects (smokers: $n=26$ ) were enrolled in our study. Smoking habits were self-reported. We excluded subjects with a history of coronary diseases and the use of antioxidative, anti-hypertensive, anti-diabetic and lipid-lowering drugs. The Ethics Committee approved the study (No. 18-102, 19-rev17), and the subjects gave their informed consent. In fasted serum samples, lipid profiles were enzymatically measured, and oxHDL was measured using an enzyme-linked immunosorbent assay (Hoken-Kagaku West, Co. Ltd., Kyoto, Japan) [1]. A $t$-test and $\chi^{2}$ test were performed for assessments between the groups, and a general linear analysis for oxHDL with adjustment for variables was also used. $P<0.05$ was considered significant.

Table I shows the clinical characteristics of the subjects studied. Smokers had a lower level of total and HDL-cholesterol and a higher level of oxHDL and oxHDL/HDL-cholesterol than non-smokers. The difference in the oxHDL or oxHDL/HDL-cholesterol levels between smokers and non-smokers remained significant after adjusting for the age, gender and body mass index (oxHDL, $p=0.01$; oxHDL/HDL-cholesterol, $p<0.01$ ).

We thus detected high oxHDL levels in smokers. This finding is reasonable as smoking is assumed to oxidise lipoproteins, including HDL [4-6], and it is noteworthy to add the present finding to those of earlier

\author{
Corresponding author: \\ Prof. Kazuhiko Kotani \\ Division of Community \\ and Family Medicine \\ Jichi Medical University \\ 3311-1 Yakushiji \\ 329-0498 Shimotsuke-City \\ Japan \\ Phone: +81 285587394 \\ E-mail: kazukotani@jichi.ac.jp
}


Table I. Clinical characteristics of studied subjects

\begin{tabular}{|lccc|}
\hline Variables & Non-smokers $(n=64)$ & Smokers $(n=26)$ & $P$-value \\
\hline Age [years] & $55 \pm 15$ & $56 \pm 12$ & 0.79 \\
\hline Men, $n(\%)$ & $35(55)$ & $13(50)$ & 0.69 \\
\hline Body mass index $\left[\mathrm{kg} / \mathrm{m}^{2}\right]$ & $24.2 \pm 3.7$ & $24.5 \pm 4.4$ & 0.77 \\
\hline Total cholesterol $[\mathrm{mmol} / \mathrm{l}]$ & $5.29 \pm 0.94$ & $4.68 \pm 0.99$ & 0.01 \\
\hline Triglyceride $[\mathrm{mmol} / \mathrm{l}]$ & $1.16(0.80-1.57)$ & $1.29(0.88-2.01)$ & 0.22 \\
\hline HDL-cholesterol $[\mathrm{mmol} / \mathrm{l}]$ & $1.51 \pm 0.44$ & $1.23 \pm 0.44$ & $<0.01$ \\
\hline OxHDL $[\mathrm{ng} / \mathrm{ml}]$ & $164(111-252)$ & $215(176-513)$ & 0.02 \\
\hline OxHDL/HDL-cholesterol & $2.89(1.88-4.81)$ & $4.85(2.80-14.3)$ & $<0.01$ \\
\hline
\end{tabular}

$H D L$ - high-density lipoprotein, OxHDL - oxidised HDL. Characteristic data are shown as the mean \pm standard deviation, median (interquartile range) or subject number (\%). Triglyceride, oxHDL and oxHDL/HDL-cholesterol were calculated after log-transformation because of their skewed deviations. Significance (non-smokers versus smokers): $p<0.05$.

studies, given the limited information available concerning the relationship between oxHDL and smoking [4-6].

However, the high oxHDL level in smokers appeared to conflict with the findings of an earlier study [6]. This discrepancy might be due in part to the difference in the population (younger people in the earlier study [6] than in the present study) and/or the methodology for measuring oxHDL markers. The measurements are of concern as various kinds of markers for HDL oxidation are currently being considered. Unlike the marker used in the earlier study [6], the oxHDL marker used in the present study reacts specifically with the oxidised apolipoprotein A-1, not native HDL [2]. Which markers are most suitable for assessing the relationship between HDL and smoking status should be explored in further studies. In addition, like the marker used in the present study, the easy measurement of oxHDL with a simple assay is also an important point that should be explored in further studies on this topic.

This study was a preliminary one with certain limitations. For example, the studied population was relatively small. In addition, the cross-sectional design did not discuss the causality. The smoking habit was self-reported, and the details concerning the smoking habit (e.g. pack-year, intensity) were not examined. These points will be addressed in future research.

In conclusion, the present study suggested that smokers have high levels of oxHDL. An oxHDL marker may be a potent target for evaluating the smoking-cardiovascular disease connection.

\section{Acknowledgments}

This work was partly supported by MEXT KAKENHI Grant (No. JP 19K07872).

\section{Conflict of interest}

The authors declare no conflict of interest.

\section{References}

1. He BM, Zhao SP, Peng ZY. Effects of cigarette smoking on HDL quantity and function: implications for atherosclerosis. J Cell Biochem 2013; 114: 2431-6.

2. Kotani K, Sakane N, Ueda M, et al. Oxidized high-density lipoprotein is associated with increased plasma glucose in non-diabetic dyslipidemic subjects. Clin Chim Acta 2012; 414: 125-9.

3. Rysz-Górzyńska M, Banach M. Subfractions of high-density lipoprotein (HDL) and dysfunctional HDL in chronic kidney disease patients. Arch Med Sci 2016; 12: 844-9.

4. Park KH, Shin DG, Cho KH. Dysfunctional lipoproteins from young smokers exacerbate cellular senescence and atherogenesis with smaller particle size and severe oxidation and glycation. Toxicol Sci 2014; 140: 16-25.

5. Chen HY, Li SC, Chen LF, Wang W, Wang Y, Yan XW. The effects of cigarette smoking and smoking cessation on high-density lipoprotein functions: implications for coronary artery disease. Ann Clin Biochem 2019; 56: 100-11.

6. Kresanov P, Ahotupa M, Vasankari T, et al. The associations of oxidized high-density lipoprotein lipids with risk factors for atherosclerosis: the Cardiovascular Risk in Young Finns Study. Free Radic Biol Med 2013; 65: 1284-90. 